\title{
Differential Regulation of Starch-synthetic Gene Expression in Endosperm Between Indica and Japonica Rice Cultivars
}

Tsuyoshi Inukai

\begin{abstract}
Background: Grain filling rates (GFRs) of indica rice cultivars are often higher than those of japonica cultivars. Although GFR is mainly determined by the starch accumulation rate (SAR) in endosperm, the genetic basis for SAR during the ripening period has not been well studied in rice. To elucidate the factors influencing the differing SARs between typical indica and japonica cultivars, we focused on differences in sink potentials, especially on starch synthesis in the endosperm.

Results: SAR in indica rice cultivar IR36 was significantly higher than in japonica cultivar T65. Although enzymes for both amylose and amylopectin syntheses had higher activity in IR36, amylopectin synthesis was seemingly more important for accelerating SAR because an elevation of amylose synthesis ability alone in the T65 genetic background did not result in the same level of SAR as IR36. In IR36, most starch-synthetic genes (SSGs) in the endosperm were more highly expressed during ripening than in T65. In panicle culture experiments, the SSGs in rice endosperm were regulated in either sucrosedependent or -independent manners, or both. All SSGs except SSI and BElla were responsive to sucrose in both cultivars, and GBSSI, AGPS2b and PUL were more responsive to sucrose in IR36. Interestingly, the GBSSI gene $\left(W x^{a}\right)$ in IR36 was highly activated by sucrose, but the GBSSI gene $\left(W x^{b}\right)$ in T65 was insensitive. In sucrose-independent regulation, AGPL2, SSIIIa, BEI, BEIII and ISAI genes in IR36 were upregulated 1.5 to 2 times more than those in T65. Additionally, at least SSI and BElla might be regulated by unknown signals; that regulation pathway should be more activated in IR36 than T65.

Conclusions: In this study, at least three regulatory pathways seem to be involved in SSG expression in rice endosperm, and all pathways were more active in IR36. One of the factors leading to the high SAR of IR36 seemed to be an increase in the sink potential.
\end{abstract}

Keywords: Starch accumulation rate, Amylose, Amylopectin, Sucrose, Sugar signal

\section{Background}

Starch in rice endosperm is synthesized via the coordinated activities of several enzymes (Jeon et al. 2010). ADP-glucose, serving as the glucose donor for starch synthesis, is mainly synthesized in cytoplasm by the ADP-glucose pyrophosphorylase (AGPase), that is a heterotetramer consisting of two small subunits (AGPS2b) and two large subunits (AGPL2). After ADP-glucose is transported from the cytoplasm to amyloplasts, glucan chains with a certain degree of polymerization are first synthesized as primers for starch synthesis by plastidial starch phosphorylase (Pho1) (Satoh et al. 2008). Starch consists of two types of glucan polymers: amylose and

Correspondence: yoshi@abs.agr.hokudai.ac.jp

Research Faculty of Agriculture, Hokkaido University, Sapporo 060-8589, Japan amylopectin. Amylose comprises predominantly linear chains of $\alpha(1-4)$-linked glucose residues and is synthesized by granule-binding starch synthase I (GBSSI) encoded by the $W x$ gene (Jeon et al. 2010). While the wild type allele $W x^{a}$ is found in most rice cultivars belonging to indica subspecies, the mutant allele $W x^{b}$ is widely distributed in japonica subspecies (Sano 1984; Sano et al. 1991). $W x^{b}$ possesses a $G$ to $T$ mutation at the $5^{\prime}$ splicing site of the first intron, which leads to a decrease in the splicing efficiency (Bligh et al. 1998; Cai et al. 1998; Isshiki et al. 1998; Hirano et al. 1998). Therefore, the GBSSI activity of japonica is considerably weak and results in starch with a low amylose content. Amylopectin has a multiple cluster structure consisting of a highly branched glucan with $\alpha$-1,6-glucosidic bonds (Jeon et al. 2010), and its synthesis is coordinately 
catalyzed by three classes of enzymes: soluble starch synthases (SSs: SSI, SSIIa and SSIIIa), starch branching enzymes (BEs: BEI, BEIIa, BEIIb) and starch debranching enzymes (isomerase 1 [ISA1] and pullulanase [PUL]) (Jeon et al. 2010). While SSs catalyze the elongation reaction of $\alpha(1-4)$-linked glucose residues, BEs introduce $\alpha-1,6$-glucosidic bond to them. ISA1 and PUL remove unnecessary $\alpha-1,6$-glucosidic bonds that interfere with formation of normal amylopectin clusters. For SSIIa, four amino acid (AA) substitutions exist between the indica and japonica cultivars (Nakamura et al. 2005); two of these substitutions are in the C-terminal region and are crucial for the SSIIa activity. Thus, the japonica cultivars lost almost all SSIIa activity, resulting in significant differences in the short to medium chain ratio within amylopectin clusters (Nakamura et al. 2005).

Important transcription factors that regulate the starch-synthetic genes (SSGs) have been identified in rice. RSR1 is a negative regulator of the SSGs in the endosperm, and the expression of all SSGs was upregulated in mutant $r s r 1$, resulting in larger grains and higher grain weight and amylose content (Fu and Xue 2010). Alkaline leucine zipper transcription factor OsbZIP58 directly regulates $A G P L 3, W x, S S I I a, B E I, B E I I b$ and ISA2 in a positive manner (Wang et al. 2013). In osbZIP58 mutants, starch and amylose contents were significantly lower than in the wild type. On the other hand, some SSGs such as the $W x$ and BEIIb genes are temperature-responsive (Hirano and Sano 1998; Yamakawa et al. 2007), and the promoter of the $W x^{b}$ gene is responsive to cool temperatures (Hirano and Sano 1998). For transcriptional regulation of $W x^{b}$, loci $d u-1$ and $d u-2$ might be involved as splicing factors in alternative splicing of premRNA of $W x^{b}$ (Isshiki et al. 2000).

The signaling pathway controlling starch synthesis in rice endosperm remains unclear. The expression level of plastidial $A G P L 3$ is synergistically regulated by both sucrose and abscisic acid (ABA) in cultured cells of rice (Akihiro et al. 2005). Recently, ZmSSIIIa, a maize homolog of the rice SSIIIa, was shown to be positively modulated by the ZmEREB156 transcription factor with synergistic regulation by sucrose and $A B A$ (Huang et al. 2016).

Early maturation is one of the most important traits in rice breeding, especially in temperate regions where the optimum season for rice cultivation is often limited. Because the main target in breeding for maturity is the time required for heading, many genes for heading time have been cloned and the regulatory networks clarified (Tsuji et al. 2013; Matsubara et al. 2014; Shrestha et al. 2014). Although the grain-filling rate (GFR) during ripening also affects maturity, GFR is rarely a breeding target because the genetic basis for GFR is not well understood. In indica, genetic variation in GFR has often been reported to be higher than in japonica (Nagato and Chaudhry 1969; Yoshida and Hara 1977; Osada et al. 1983). Multiple factors such as photosynthesis activity in source organs, efficiency of sugar translocation and/or starch synthesis activity in sink organs appear to be involved in the difference in GFR between the two subspecies. However, Murchie et al. (2002) reported that the differences of GFR among rice cultivars is not explained by differences in source properties such as lightsaturated rate of photosynthesis or in the level of ribulose 1,5 bisphosphate carboxylase oxygenase or total chlorophyll. Because differences in the sink potential, especially in the ability to synthesize starch, among rice cultivars have not been thoroughly studied, here we compared sink potentials between typical indica and japonica rice cultivars in terms of SSG regulation and found that the SSGs in endosperm were differentially regulated between the two rice cultivars.

\section{Results \\ Differential Regulation of Starch-synthetic Gene Expression Between IR36 and Taichung 65 (T65)}

In this study to compare indicators of starch accumulation in karyopses between indica and japonica rice cultivars under the same environmental conditions, indica cultivar IR36 and japonica cultivar T65 were selected because their vegetative growth in a greenhouse at Hokkaido University in Sapporo during the summer differed by only several days. To sample the spikelets flowering at the same time, sowing dates of those cultivars were adjusted. The mean temperature in the greenhouse during the summer was always over $25{ }^{\circ} \mathrm{C}$, suitable for tropical cultivars IR36 and T65.

Both the dry mass of the karyopsis and the amount of starch in the endosperm of IR36 peaked (16.1 and 13.0 mg karyopsis ${ }^{-1}$ ) about 1 week earlier than in T65 (21.3 and $17.3 \mathrm{mg}$ karyopsis $^{-1}$ ) (Fig. 1a and d). The difference in grain weight between IR36 and T65 was relatively large, suggesting that the number of endosperm cells also differed significantly between the two cultivars because the grain weight is strongly correlated with the number of endosperm cells in rice (Yang et al. 2002). Because GFR and the starch accumulation rate (SAR) per cell in IR36 would be underestimated if the differences in GFR and SAR between IR36 and T65 were compared using the absolute values of grain weight and starch content, the relative values for each final weight were used to compare GFR and SAR between the two cultivars (Fig. 1b, c, e and f). As shown in Fig. 1c and f, GFR and SAR in IR36 were significantly higher than in T65 from 8 to 14 days after flowering (DAF). Thus, IR36 seemed to be able to synthesize endosperm starch faster than T65, resulting in early maturation of IR36. 

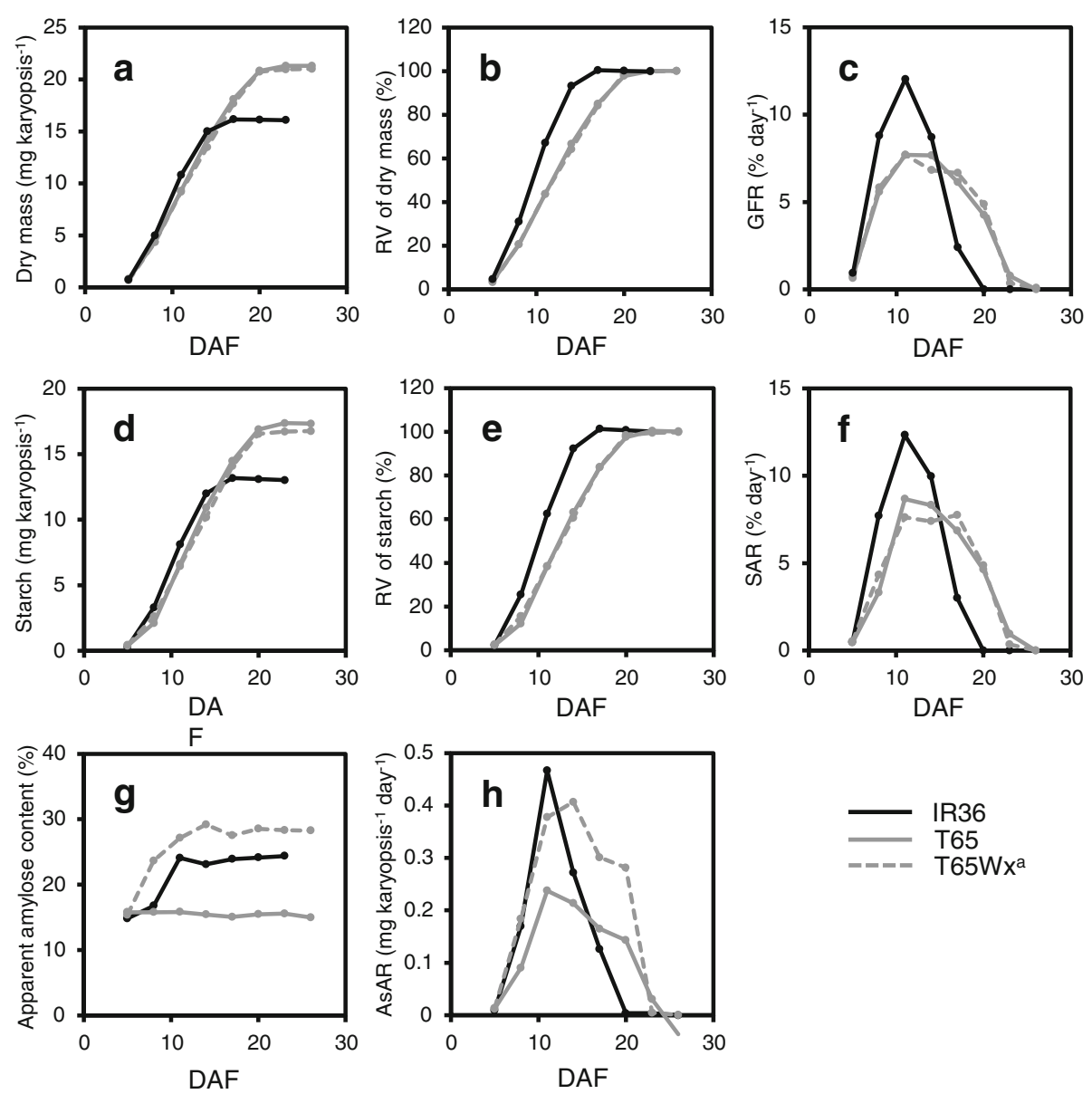

Fig. 1 Starch accumulation in rice endosperm of indica cultivar IR36, japonica cultivar T65 and T65 near-isogenic line T65W $x^{\mathrm{a}}$ over time during ripening. a Dry mass. b Relative value (RV) of dry mass. c Grain filling rate (GFR) calculated from data in panel b. d Starch content. e RV of starch content. f Starch accumulation rate (SAR) calculated from data in panel e. $\mathbf{g}$ Apparent amylose content. $\mathbf{h}$ Amylose accumulation rate (AsAR) calculated from data in panels $\mathbf{d}$ and $\mathbf{g}$. DAF, days after flowering

To elucidate the factors responsible for the difference in SAR between IR36 and T65, we first investigated the relationship between SAR and the activity of the amylose-synthesizing enzyme GBSSI. Because IR36 has the wild-type allele $W x^{a}$ and T65 has the mutant allele $W x^{b}$ and thus lower amylose synthesis, the difference in SAR could be due to this difference in amylose synthetic activity. Indeed, $W x^{a}$ gene expression level and GBSSI activity in IR36 was about $4 \times$ and $10 \times$ higher, respectively, than in T65 at 10 DAF (Figs. 2, 3). Thus, the amylose accumulation rate (AsAR) was higher in IR36 than in T65 (Fig. 1h). To determine whether this difference in AsAR affected SAR, the SAR in a T65 near-isogenic line carrying $W x^{a}\left(\mathrm{~T} 65 \mathrm{Wx} \mathrm{x}^{\mathrm{a}}\right)$ was compared with that of $\mathrm{T} 65$. As in IR36, T65W $\mathrm{x}^{\mathrm{a}}$ had high $W x^{a}$ expression, GBSSI activity and AsAR (Figs. 1h, 2, 3); however, the SAR in T65Wx ${ }^{\mathrm{a}}$ was similar to T65 and thus lower than in IR36 (Fig. 1f). These results indicated that elevation of amylose synthetic ability alone is not sufficient for the increase in SAR in rice endosperm, probably because enzymes for the synthesis of amylose and for amylopectin compete for the same substrate, ADP-glucose. Thus, the high SAR of IR36 appears to be due to either the high activity for the synthesis of both amylose and amylopectin or only for amylopectin.

We next compared the total activities of AGPases that synthesize ADP-glucose, a substrate for the synthesis of both amylose and amylopectin and of the SSs that are in involved in amylopectin synthesis. For the total AGPase activity, a slight but significant difference was found between IR36 and T65 at 7 DAF $(P<0.001$; Fig. 2$)$; however, the pattern of AGPase activity over time was almost similar between IR36 and T65 until IR36 had matured (Fig. 2). On the other hand, the total activity of SSs from 5 to 10 DAF was always higher in IR36 than in T65, and at the peak level at 7 DAF was about $2 \times$ higher than in T65 $(P<0.001)$ (Fig. 2$)$. This result was highly consistent with the differences in SAR observed between 


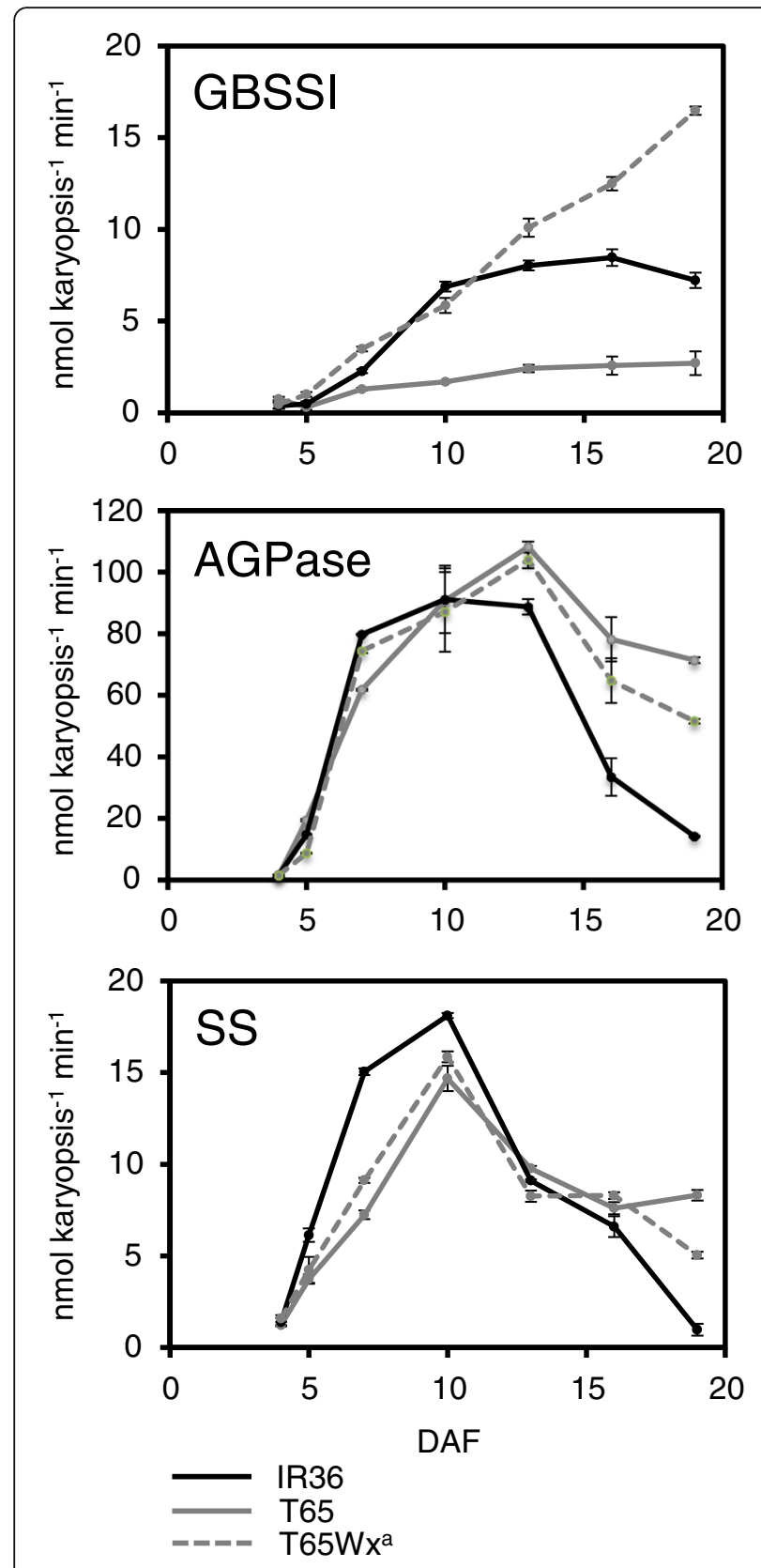

Fig. 2 Activity of ADP-glucose pyrophosphorylase (AGPase), granulebound starch synthase I (GBSSI) and starch synthases (SSs) in endosperm of rice cultivars IR36, T65 and T65W $x^{a}$ over time during ripening. Error bars indicate the SE for biological triplicates. DAF, days after flowering

IR36 and T65 and strongly suggested that a high synthesis of amylopectin was one factor leading to the high SAR in IR36.

To analyze the factors contributing to the difference in amylopectin synthesis between IR36 and T65, we analyzed the expression patterns of the genes related to amylopectin synthesis from 4 to 13 DAF. The expression of SS, BE and DBE genes sharply increased from 7 to 10 DAF in IR36, as opposed to a rather gradual increase in
T65 (Fig. 3), so that the expression of all amylopectin synthetic genes was several-fold higher in IR36 by 10 DAF ( $P<0.05$ for all amylopectin synthetic genes; Fig. 3$)$. Although the expression of AGPS2 $b$ and AGPL2 genes was also remarkably upregulated in IR36 compared with T65 (Fig. 3), such proportional differences in the total AGPase activity between IR36 and T65 were not found (Fig. 2). The expression of Pho1, which encodes a plastidial phosphorylase, involved in the synthesis of glucan primers, was similar between IR36 and T65 (Fig. 3). Taken together, these results suggested that enzymes in the pathway for amylopectin synthesis were highly active in IR36, leading to the high SAR.

Sugar-dependent and -Independent Regulation of Starchsynthetic Gene Expression in Endosperm

To determine the regulatory pathway(s) that contribute to the differential regulation of the SSGs between IR36 and $\mathrm{T} 65$, we investigated the response patterns of each SSG to sucrose in panicles that had been harvested at 3 DAF, then cultured in water at $25^{\circ} \mathrm{C}$ for $24 \mathrm{~h}$. After panicle transfer to $0 \mathrm{mM}$ or $100 \mathrm{mM}$ sucrose and incubated at $25{ }^{\circ} \mathrm{C}$ for $24 \mathrm{~h}$, the expression of all SSGs except SSI and BEIIa in both cultivars had increased in response to $100 \mathrm{mM}$ sucrose (Fig. 4a). Only the GBSSI in IR36 $\left(W x^{a}\right)$ was responsive to sucrose, not that in T65 $\left(W x^{b}\right)$ (Fig. 4a). Thus, most SSGs in endosperm were regulated in a sucrose-dependent manner. To confirm whether $W x^{b}$ in T65 had completely lost responsiveness to sucrose, we investigated the change in expression of $W x^{b}$ and $W x^{a}$ as sucrose levels varied from 0 to $300 \mathrm{mM}$. The expression of the $W x^{a}$ genes in both IR36 and $\mathrm{T} 65 \mathrm{Wx}^{\mathrm{a}}$ increased with increasing sucrose concentration, while the expression of $W x^{b}$ in T65 was not upregulated even at $300 \mathrm{mM}$ sucrose, indicating that $W x^{b}$ was mostly insensitive to sucrose (Fig. 5). On the other hand, when the gene expression data were compared between IR36 and T65 at the $0 \mathrm{mM}$ sucrose level, the expression of AGPL2, SSIIIA, BEI, BEIIb and ISA1 were 1.5 to $2 \times$ higher in IR36 than in T65, while Pho1 expression was $2 \times$ lower in IR36 than in T65 (Fig. 4b). These data suggested that some SSGs were also regulated independently of sucrose signals. At $100 \mathrm{mM}$ sucrose, the comparative patterns of the SSGs, except GBSSI, $A G P S 2 b$ and PUL, between IR36 and T65 were almost similar to those at $0 \mathrm{mM}$ sucrose (Fig. $4 \mathrm{~b}$ ), indicating that GBSSI, AGPS2 and PUL in IR36 were more responsive to sucrose than those in T65; the response patterns of the rest of the SSGs were well conserved between IR36 and T65.

Thus, the expression of some SSGs in rice was regulated in at least two ways, namely, a sucrose-dependent and a sucrose-independent manner. In both regulatory modes, distinct differences were found between IR36 

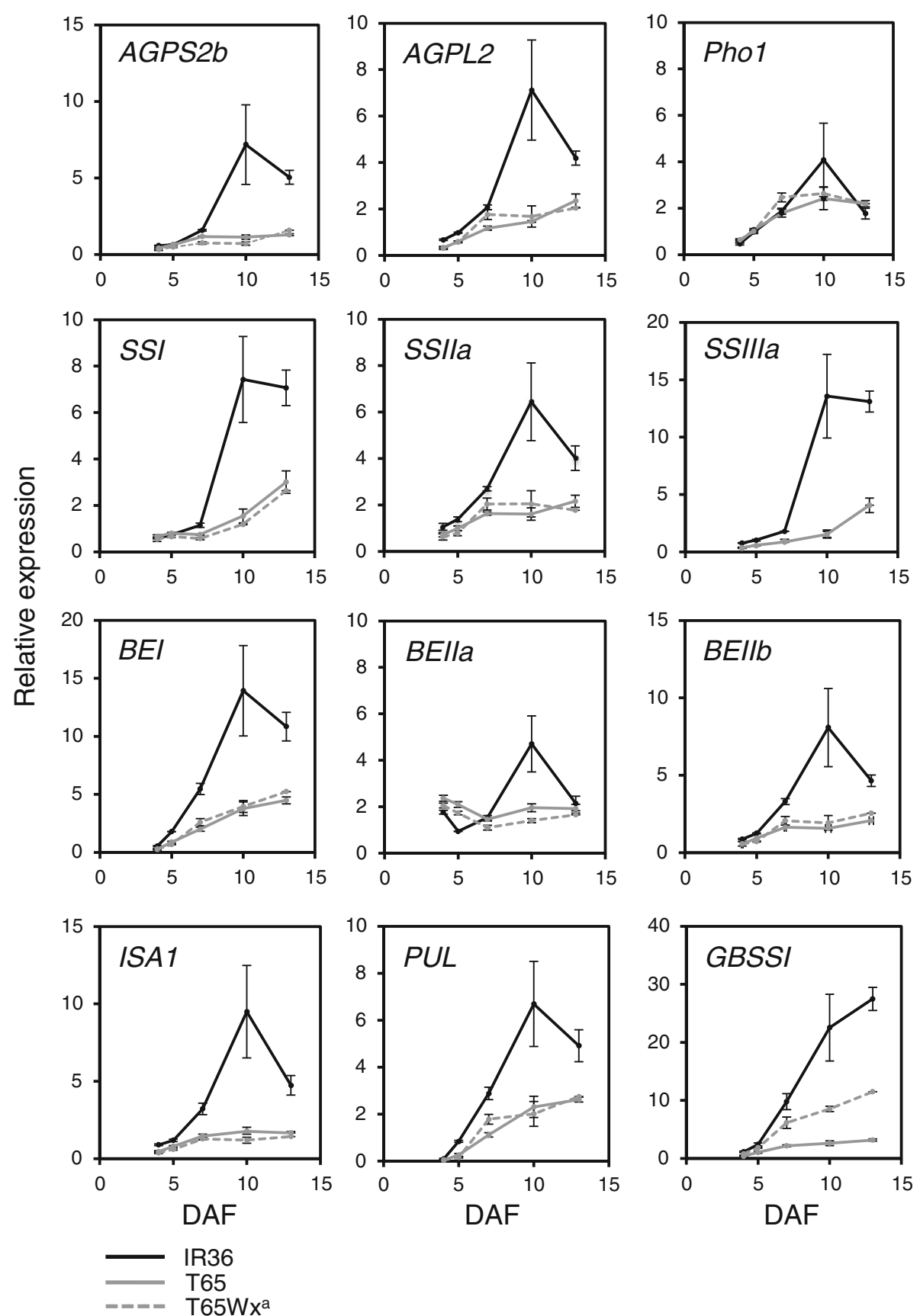

Fig. 3 Relative transcript levels of genes involved in starch synthesis in endosperm over time during ripening period among rice cultivars IR36, T65 and T65Wxa . The relative ratios were calculated using the geometric mean of the four internal standard genes actin1, eEF-1a, elF-4a and a-tubulin. Error bars indicate the SE for biological triplicates. DAF, days after flowering

and T65. The expression level of SSI and BEIIa in the cultured panicles was almost similar between IR36 and T65, regardless of the sucrose concentration (Fig. 4). However, because the expression of both genes was considerably upregulated in IR36 than T65 at 10 and/or 14 DAF under typical growth conditions (Fig. 3), an unknown pathway(s) might be still involved in regulating the expression of the SSGs in rice endosperm.
In the sucrose-dependent regulation of SSGs, those expression levels appeared to be determined by the sucrose concentration in the endosperm cells. When we assayed the sucrose concentration in a crude extract of developing karyopses during ripening, the sucrose concentration in IR36 was mostly constant at about $120 \mathrm{mM}$, while the sucrose level in T65 was lower (70-90 mM) from 4 to 5 DAF and only reached $100 \mathrm{mM}$ by 7 DAF (Fig. 6). 


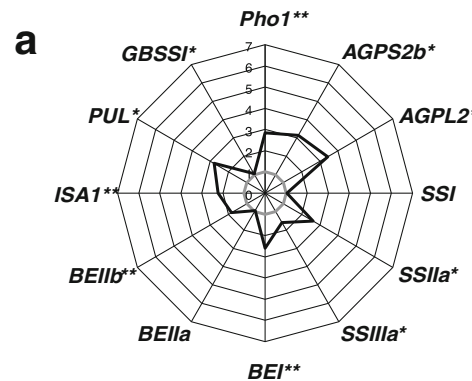

T65

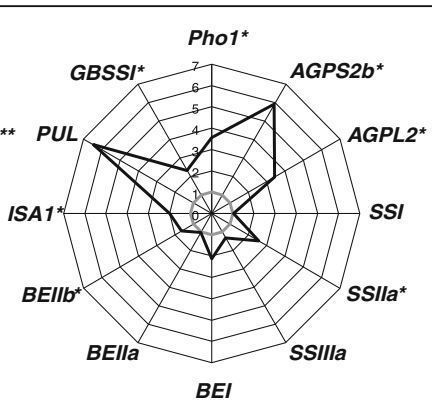

IR36

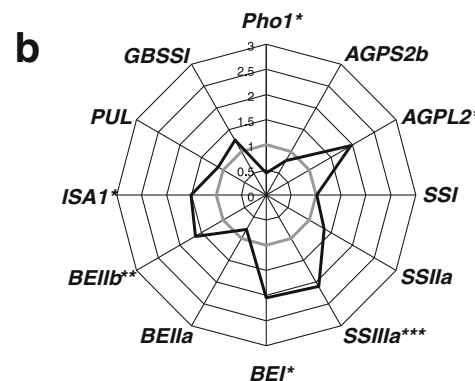

$0 \mathrm{mM}$ sucrose

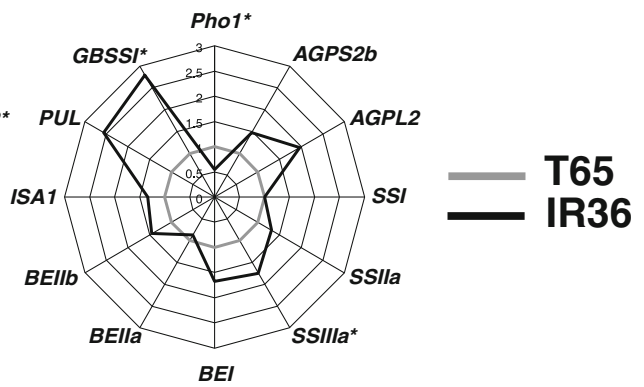

$100 \mathrm{mM}$ sucrose

Fig. 4 Expression profiles for starch-synthetic genes in endosperm in cultured panicles at 5 DAF of rice indica cultivar IR36 and japonica cultivar T65. $\mathbf{a}$ Effect of 0 and $100 \mathrm{mM}$ sucrose on gene expression in each cultivar. Expression is given relative to the value at $0 \mathrm{mM}$ sucrose. $\mathbf{b}$ Differences in gene expression between IR36 and T65 exposed to 0 or $100 \mathrm{mM}$ sucrose. Significant differences were determined using biological triplicates and Student's $t$-test $\left({ }^{*} P \leq 0.05,{ }^{* *} P \leq 0.01,{ }^{* * *} P \leq 0.001\right)$

These results suggested that the differences of the SSG expression between IR36 and T65 might also be indirectly caused through sucrose-dependent regulation.

\section{Discussion}

In our analyses of the difference in SAR between indica and japonica rice cultivars from the viewpoint of sink potentials, the higher starch accumulation in IR36 mainly depended on greater amylopectin synthesis; most

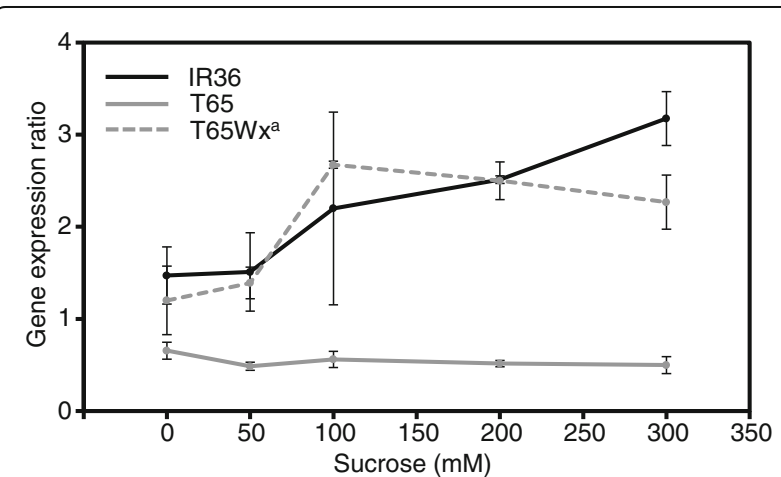

Fig. 5 Effects of different sucrose concentrations on expression of $W x^{a}$ and $W x^{b}$ genes. Error bars indicate the SE for biological triplicates. DAF, days after flowering genes involved in amylopectin synthesis were highly upregulated in IR36. The SSGs were regulated in either a sucrose-dependent or -independent manner, or both, and other regulation pathways might also be involved in the expression of SSGs such as SSI and BEIIa. In IR36, all these regulatory systems for amylopectin synthesis were more active than in T65.

Although some SSGs were regulated by multiple systems, which regulatory systems were most crucial for

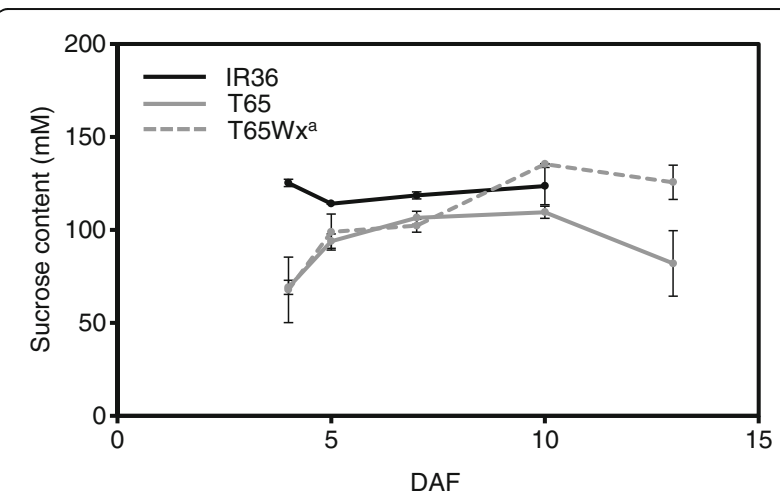

Fig. 6 Sucrose content in crude extracts of developing karyopses of IR36, T65 and T65W $x^{a}$ during ripening. Error bars indicate the SE for biological triplicates. DAF, days after flowering 
achieving the higher expression of those SSGs in IR36 was unclear. In panicle culture without sucrose, AGPL2, SSIIIa, BEI, BEIIb and ISA1 genes of IR36 were more upregulated than in T65 (Fig. 4b). This sucroseindependent regulation appeared to define the basal level of the SSG expression; these SSG levels increased further in a sucrose-dependent manner (Fig. 4a). Although the sucrose-response patterns of most SSGs for amylopectin synthesis were similar between IR36 and T65 (Fig. 4), this sucrose-dependent regulation could also indirectly contribute to the high SSG expression in IR36. Actually, the sucrose concentration in crude extracts from developing seeds of IR36 was higher than in those of T65 in the early to middle phase of ripening (Fig. 6), so expression of the sucrose-responsive SSGs was expected to be higher in IR36 than in T65 during that period.

In this study, we did not obtain any information on the regulation of the SSI and BEIIa genes. For these genes, unknown signals might be involved in the regulation of their expression. For instance, Akihiro et al. (2005) reported that the expression of the plastidial AGPL3 gene was significantly enhanced by exogenous application of ABA to rice suspension culture cells in the presence of sucrose. Interestingly, only $A B A$ treatment decreased the expression of OsAPL3 (Akihiro et al. 2005). These facts suggest that not only ABA but also the interaction of ABA with a sucrose signal are important to activate expression of APGL3. However, neither SSI nor BEIIa were upregulated by $10-100 \mathrm{mM}$ ABA plus $100 \mathrm{mM}$ sucrose in our preliminary results (unpublished data). Studies on the involvement of other hormone signals and/or their synergistic effects with sucrose signals in starch synthesis are needed to better understand the regulation of SSGs in rice endosperm.

Sugars function as signal molecules in plant development, growth and responses to environmental stresses (Rolland et al. 2006; Eveland and Jackson 2012; Lastdrager et al. 2014). Sugar signals, as we have shown here, apparently also play important roles in defining source-sink relationships in rice. The sink potential of rice endosperm is partly determined by the amount of translocated sugar supplied from the source organs; sink strength is always coordinated with the strength of the source such as the productivity of photosynthesis in leaves and/or the efficiency of sugar translocation through phloem. It is noteworthy that the sucrose-dependent regulation was not uniform among genes for amylose and amylopectin syntheses, suggesting that any fluctuation in sucrose translocation may affect amylopectin structure and/or the ratio of amylopectin to amylose.

We found a distinct difference in sucrose responsiveness between the $W x^{a}$ and $W x^{b}$ alleles (Figs. 4 and 5). $W x^{a}$ was highly responsive to sucrose while the sucrose responsiveness in $W x^{b}$ appeared to be almost lost. So far, the difference in the expression levels between $W x^{a}$ and $W x^{b}$ has mainly been explained by a decline in splicing efficiency caused by the base substitution at the splicing site of intron 1 of $W x^{b}$ (Bligh et al. 1998; Cai et al. 1998; Isshiki et al. 1998; Hirano et al. 1998). However, other factors such as a difference in sucrose responsiveness could also be involved in the differential regulation in the $W x$ gene. Because the sucrose-response pattern of $W x^{a}$ in $\mathrm{T} 65 \mathrm{Wx}^{\mathrm{a}}$ was similar to that in IR36, the difference in the sucrose response between $W x^{a}$ and $W x^{b}$ might be due to the differences in the cis-acting regulatory sequences.

Because T65 possessed the alk allele at the Alk locus encoding SSIIa (data not shown), SSIIa enzyme activity in T65 should be nearly lost after the substitution a few amino acids (Nakamura et al. 2005). Therefore, the lower SSs activity in T65 compared with IR36 might be due not only to a reduction in the level of SSGs but also to a decline in SSIIa activity. To elucidate to what extent each enzyme encoded by the SSGs, including SSIIa, is rate-limiting for starch synthesis in the endosperm, we need to develop and analyze a series of IR36 mutants for each SSG.

During ripening, the AGPase activity between IR36 and T65 differed little although the expression of both $A G P S 2 b$ and AGPL2 genes was much higher in IR36 than in T65. In rice endosperm, AGPase is positively regulated by 3-phosphoglyceric acid (3-PGA) and negatively by inorganic phosphate (Pi) (Sikka et al. 2001; Sakulsingharoj et al. 2004; Tuncel et al. 2014). Although it is still uncertain how 3-PGA and Pi can signal the availability of carbon and energy for starch synthesis in the endosperm, the cytosolic AGPase activity in rice endosperm might be maintained at a certain level due to such allosteric regulation although the expression levels of $A G P S 2 b$ and AGPL2 fluctuated. We also showed that the cytosolic $A G P S 2 b$ and $A G P L 2$ genes were highly upregulated at $100 \mathrm{mM}$ sucrose in endosperms of both IR36 and T65. However, such high responsiveness to sucrose in those genes was not observed in suspension culture cells of japonica cultivar Nipponbare (Akihiro et al. 2005). These differences suggest that the expression of the AGPS2b and AGPL2 genes might be under tissuespecific regulation or vary among japonica cultivars.

The SAR did not significantly increase in $\mathrm{T} 65 \mathrm{Wx} \mathrm{x}^{\mathrm{a}}$ with high amylose synthesis. When only amylose synthesis activity increased, why was SAR not elevated? Can the activation of both amylose and amylopectin synthesis increase SAR? According to Martin and Smith (1995), amylopectin synthesis begins before amylose synthesis and that amylose is later synthesized within developing starch granules because GBSSI is confined inside the starch granule by its own binding to the granule. Therefore, inside developing granules, enzymes for the 
amylose and amylopectin syntheses could equally compete for the same substrate, but on the surfaces of developing granules, amylopectin synthesis might proceed preferentially and not compete with amylose synthesis. For such reasons, activation of only amylose synthesis is not considered to be responsible for the higher SAR in IR36. For high SAR in rice endosperm, higher amylopectin synthesis activity seems to be essential.

Although the expression level of SSI in IR36 was much higher than in T65 during ripening, Takemoto-Kuno et al. (2006) reported that the SSI expression in the indica cultivar Kasalath was lower than in the japonica cultivar Nipponbare. These facts suggest that the regulation of SSGs may be quite variable within and between subspecies. Because TFs of SSGs in rice, RSR1 and OsbZIP58, were previously reported and their target SSGs were characterized (Fu and Xue 2010; Wang et al. 2013), we know that RSR1 targets all SSGs, whereas only some of the SSGs are targeted by OsbZIP58. Functional variation in such master regulators of SSGs might be one factor leading to the diversification of the SSG expression pattern in rice endosperm.

\section{Conclusions}

In this study of potential sink factors leading to the high SAR in IR36, we showed that a high level of amylopectin synthesis was crucial for the high starch synthesis in IR36. The SSG regulatory systems in the rice endosperm are rather complicated; at least three pathways are probably involved in the signaling to activate SSG expression. At the basal level, the SSGs in IR36 seemed to be more highly expressed than in T65, and IR36 expression levels increased more due to a sucrose-dependent pathway and/or pathways involved in unknown signals. Although we did not deal here with varietal differences in source strength, the sucrose concentration in the karyopsis tissues of IR36 appeared to be maintained at a higher level than in T65, especially during early to mid ripening, suggesting that the source strength of IR36 was more reinforced than T65. Thus, the high SAR in IR36 appears to be achieved by a well-coordinated balance of source supply and sink demand.

\section{Methods}

\section{Plant Materials and Growth Conditions}

Rice cultivar IR36 (subsp. indica) and T65 (subsp. japonica) were used in this study. The T65 near-isogenic line (NIL) carrying the $W x^{a}$ gene, $\mathrm{T} 65 \mathrm{Wx}^{\mathrm{a}}$, was also used (Mikami et al. 1999). Seeds of T65 Wx ${ }^{a}$ were obtained through the courtesy of Dr. Y. Sano, Graduate School of Agriculture, Hokkaido University. For measuring amylose, starch from two amylose-free lines T65wx and TR60 were used as standards for amylopectin. T65wx is a T65 NIL carrying $w x$ (Mikami et al. 1999);
TR60 was a $\mathrm{F}_{3}$ line derived from the cross between T65wx and IR36 and possesses $w x$ and Alk. Plants were grown in the greenhouse of Hokkadio University at Sapporo from April to August. Sowing dates for IR36, $\mathrm{T} 65$ and $\mathrm{T} 65 \mathrm{Wx}{ }^{\mathrm{a}}$ were adjusted so that spikelets from IR36, T65 and T65W $\mathrm{x}^{\mathrm{a}}$ for all experiments were flowering at the same time.

\section{Panicle Culture}

Rice panicles were cultured by the method of Hirano and Sano (1998). Briefly, rice panicles with the stem were sampled $3 \mathrm{DAF}$, and samples were then cut at the node just beneath the panicle with a razor in water. The panicle separated from the stem was immediately transferred to a test tube including $5 \mathrm{ml}$ of water and covered with a plastic bag to prevent drying. After $24 \mathrm{~h}$, the panicle was transferred to another test tube with $5 \mathrm{ml}$ of water or $100 \mathrm{mM}$ sucrose solution and incubated for $24 \mathrm{~h}$. The sucrose concentration in the culture medium was determined by previously described methods for rice (Hirano and Sano 1998; Lee et al. 2000; Kobata et al. 2001). Developing karyopses for expression analyses were then carefully excised with forceps.

\section{Measurement of Dry Mass, Starch, Amylose and Sucrose}

Spikelets that flowered at the same time were marked with a water-based marker, and 20-30 developing karyopses per cultivar were collected at a certain interval. For measuring dry mass, samples were kept in an aluminum can and dried at $105^{\circ} \mathrm{C}$. After $12 \mathrm{~h}$, they were cooled to room temperature in a dessicator, and the mass was measured. These weighed samples were then used to determine the starch content using the glucoamylase-glucose oxidase method (Thivend et al. 1965). Based on the data for dry mass and starch content, the amount of starch per karyopsis was calculated. For amylose, developing karyopses were dried at $40{ }^{\circ} \mathrm{C}$, starch granules were extracted (Yamamoto et al. 1973), and apparent amylose content was then measured using iodine colorimetry (Juliano 1971; Yamakawa et al. 2007). A starch sample from amylose-free line T65wx was used as an amylopectin standard to measure amylose content for T65 and T65Wx $\mathrm{W}^{\mathrm{a}}$. The $\mathrm{F}_{3}$ line TR60, carrying both $w x$ and $A l k$, was selected from the population derived from a cross between IR36 and T65wx and used as an amylopectin standard for IR36.

The amount of amylose per karyopsis was calculated from the starch mass per karyopsis and the amylose content. Sucrose concentration in crude extracts from developing karyopses was measured using the Sucrose Assay Kit, EnzyChrom (BioAssay Systems, Hayward, CA, USA) following the manufacturer's instructions. 


\section{Enzyme Activity Assay}

Developing karyopses were homogenized using a mortar and pestle on ice in 4-10 volumes of a grinding solution of $50 \mathrm{mM}$ HEPES-NaOH (pH 7.4), $2 \mathrm{mM} \mathrm{MgCl}_{2}$, $50 \mathrm{mM}$ 2-mercaptoethanol and $12.5 \%(\mathrm{v} / \mathrm{v})$ glycerol for assay of AGPase or $50 \mathrm{mM}$ Tris- $\mathrm{HCl}(\mathrm{pH} 7.4), 2 \mathrm{mM}$ EDTA, $5 \mathrm{mM}$ dithiothreitol, $0.4 \mathrm{mM}$ phenylmethylsulfonyl fluoride and $12.5 \%(\mathrm{v} / \mathrm{v})$ glycerol for the SS and GBSSI assays. The homogenates were centrifuged at $14,000 \mathrm{rpm}$ at $4{ }^{\circ} \mathrm{C}$ for $15 \mathrm{~min}$, and the supernatants were used as the crude enzyme extract for the AGPase and SS assays, respectively. For the GBSSI assay, the precipitated starch granules were used as the crude enzyme extract. AGPase, SS and GBSSI were assayed using the methods of Nishi et al. (2001). Three separate extracts were analyzed.

\section{Gene Expression Analysis by Quantitative RT-PCR}

Total RNA was extracted from developing karyopses using TRIzol reagent (Invitrogen, Tokyo) and the manufacturer's instructions, then treated with RNase-free DNase-I (Roche Diagnostics, Mannheim, Germany) to remove DNA contamination. The expression of the genes involved in starch synthesis of rice endosperm (Ohdan et al. 2005; Satoh et al. 2008) were assayed by the multiplex RT-PCR method using the GenomeLAB GeXP Start Kit (Beckman Coulter, Fullerton, CA, U. S. A.) as described previously (Kim et al. 2008). As internal standards, the actin1, eEF-1a, eIF-4a and $\alpha$-tubulin genes were chosen according to Li et al. (2009). Expression of the starch-synthetic genes was calculated as a relative ratio to the geometric mean of the four internal standard genes (Vandesompele et al. 2002). The sequences of the primers used in this study are summarized in Additional file 1: Table S1.

\section{Additional file}

Additional file 1: Table S1. List of primers used for expression analysis. (XLSX $31 \mathrm{~kb})$

\section{Abbreviations \\ 3-PGA: 3-phosphoglyceric acid; AA: Amino acid; ABA: Abscisic acid; AGPase: ADP- glucose pyrophosphorylase; AsAR: Amylose accumulation rate; BE: Starch branching enzyme; DAF: Days after flowering; DBE: Starch debranching enzyme; GBSSI: Granule-bound starch synthase I; GFR: Grain filling rate; ISA1: Isomerase 1; NIL: Near-isogenic line; Pho1: Plastidial starch phosphorylase; Pi: Inorganic phosphate; PUL: Pullulanase; SAR: Starch accumulation rate; SS: Starch synthase; SSG: Starch-synthetic gene; T65: Taichung 65}

\section{Acknowledgements}

The author thanks Dr. Y. Sano for providing seeds of T65W $x^{a}$ and Dr. Y. Nakamura for his valuable advise on enzyme activity assay. This work was supported in part by a grant-in-aid for scientific research on scientific research (C) from the Ministry of Education, Culture, Sports, Science and Technology.

\section{Competing Interests}

The author declares that he has no competing interests.
Received: 19 December 2016 Accepted: 21 February 2017

Published online: 28 February 2017

\section{References}

Akihiro T, Mizuno K, Fujimura T (2005) Gene expression of ADP-glucose pyrophosphorylase and starch contents in rice cultured cells are cooperatively regulated by sucrose and ABA. Plant Cell Physiol 46(6):937-46

Bligh HFJ, Larkin PD, Roach PS, Jones CA, Fu H, Park WD (1998) Use of alternate splice sites in granule-bound starch synthase mRNA from low-amylose rice varieties. Plant Mol Biol 38(3):407-15

Cai XL, Wang ZY, Xing YY, Zhang JL, Hong MM (1998) Aberrant splicing of intron 1 leads to the heterogeneous 5' UTR and decreased expression of waxy gene in rice cultivars of intermediate amylose content. Plant J 14(4):459-65

Eveland AL, Jackson DP (2012) Sugars, signaling, and plant development. J Exp Bot 63(9):3367-77

Fu FF, Xue HW (2010) Coexpression analysis identifies rice starch regulator1, a rice $A P 2 / E R E B P$ family transcription factor, as a novel rice starch biosynthesis regulator. Plant Physiol 154(2):927-38

Hirano HY, Sano Y (1998) Enhancement of Wx gene expression and the accumulation of amylose in response to cool temperatures during seed development in rice. Plant Cell Physiol 39(8):807-12

Hirano HY, Eiguchi M, Sano Y (1998) A single base change altered the regulation of the Waxy gene at the posttranscriptional level during the domestication of rice. Mol Biol Evol 15(8):978-87

Huang H, Xie S, Xiao Q, Wei B, Zheng L, Wang Y, Cao Y, Zhang X, Long T, Li Y, Hu Y, Yu G, Liu H, Liu Y, Huang Z, Zhang J, Huang Y (2016) Sucrose and ABA regulate starch biosynthesis in maize through a novel transcription factor, ZmEREB156. Sci Rep 6:27590

Isshiki M, Morino K, Nakajima M, Okagaki RJ, Wessler SR, Izawa T, Shimamoto K (1998) A naturally occurring functional allele of the rice waxy locus has a GT to $\Pi$ mutation at the $5^{\prime}$ splice site of the first intron. Plant J 15(1):133-8

Isshiki M, Nakajima M, Satoh H, Shimamoto K (2000) dull: rice mutants with tissue-specific effects on the splicing of the waxy pre-mRNA. Plant J 23(4): 451-60

Jeon JS, Ryoo N, Hahn TR, Walia H, Nakamura Y (2010) Starch biosynthesis in cereal endosperm. Plant Physiol Biochemi 48(6):383-92

Juliano BO (1971) A simplified assay for milled-rice amylose. Cereal Sci Today 16(11):334-40

Kim B, Masuta C, Matsuura H, Takahashi H, Inukai T (2008) Veinal necrosis induced by turnip mosaic virus infection in Arabidopsis is a form of defense response accompanying HR-like cell death. Mol Plant Microbe Interact 21(2):260-8

Kobata T, Hamahara Y, Matsuyama S (2001) Liquid culturing of detached panicles of rice: cooled culture solutions extended the period of growth. Plant Prod Sci 4(4):280-2

Lastdrager J, Hanson J, Smeekens S (2014) Sugar signals and the control of plant growth and development. J Exp Bot 65(3):799-807

Lee HJ, Seebauer JR, Below FE (2000) An improved technique for culture of rice panicles. Plant Cell Tiss Org Cult 60:55-60

Li QF, Zhang GY, Dong ZW, Yu HX, Gu MH, Sun SSM, Liu QQ (2009) Characterization of expression of the OsPUL gene encoding a pullulanasetype debranching enzyme during seed development and germination in rice. Plant Physiol Biochem 47(5):351-8

Martin C, Smith AM (1995) Starch biosynthesis. Plant Cell 7(7):971-85

Matsubara K, Hori K, Ogiso-Tanaka E, Yano M (2014) Cloning of quantitative trait genes from rice reveals conservation and divergence of photoperiod flowering pathways in Arabidopsis and rice. Front Plant Sci 5:193

Mikami I, Aikawa M, Hirano HY, Sano Y (1999) Altered tissue-specific expression at the $W \times$ gene of the opaque mutants in rice. Euphytica 105(2):91-9

Murchie EH, Yang J, Hubbart S, Horton P, Peng S (2002) Are there associations between grain-filling rate and photosynthesis in the flag leaves of fieldgrown rice? J Exp Bot 53(378):2217-24

Nagato K, Chaudhry FM (1969) A comparative study of ripening process and kernel development in Japonica and Indica rice. Proc Crop Sci Soc Japan 38(3):425-33

Nakamura Y, Francisco PB Jr, Hosaka Y, Sato A, Sawada T, Kubo A, Fujita N (2005) Essential amino acids of starch synthase lla differentiate amylopectin structure and starch quality between japonica and indica rice varieties. Plant Mol Biol 58(2):213-27

Nishi A, Nakamura Y, Tanaka N, Satoh H (2001) Biochemical and genetic analysis of the effects of amylose-extender mutation in rice endosperm. Plant Physiol 127(2):459-72 
Ohdan T, Francisco PB Jr, Sawada T, Hirose T, Terao T, Satoh H, Nakamura Y (2005) Expression profiling of genes involved in starch synthesis in sink and source organs of rice. J Exp Bot 56(422):3229-44

Osada A, Ishizaki Y, Suzuki S (1983) Difference in the number of days for ripening of grains between Japonica and Indica rice cultivars. Jpn J Trop Agr 27(2):59-66

Rolland F, Baena-Gonzalez E, Sheen J (2006) Sugar sensing and signaling in plants: conserved and novel mechanisms. Annu Rev Plant Biol 57:675-709

Sakulsingharoj C, Choi S-B, Hwang S-K, Edwards GE, Bork J, Meyer CR, Preiss J, Okita TW (2004) Engineering starch biosynthesis for increasing rice seed weight: the role of the cytoplasmic ADP-glucose pyrophosphorylase. Plant Sci 167(6):1323-33

Sano Y (1984) Differential regulation of waxy gene expression in rice endosperm. Theor Appl Genet 68(5):467-73

Sano Y, Hirano HY, Nishimura M (1991) Evolutionary significance of differential regulation at the wx locus of rice. In: IRRI KSDFJSD (ed) Rice Genetics II. IRRI, Los Banos, pp 11-20

Satoh H, Shibahara K, Tokunaga T, Nishi A, Tasaki M, Hwang SK, Okita TW, Kaneko N, Fujita N, Yoshida M, Hosaka Y, Sato A, Utsumi Y, Ohdan T, Nakamura Y (2008) Mutation of the plastidial alpha-glucan phosphorylase gene in rice affects the synthesis and structure of starch in the endosperm. Plant Cell 20(7):1833-49

Shrestha R, Gomez-Ariza J, Brambilla V, Fornara F (2014) Molecular control of seasonal flowering in rice, arabidopsis and temperate cereals. Ann Bot 114(7): $1445-58$

Sikka VK, Choi S-B, Kavakli IH, Sakulsingharoj C, Gupta S, Ito H, Okita TW (2001) Subcellular compartmentation and allosteric regulation of the rice endosperm ADPglucose pyrophosphorylase. Plant Sci 161(3):461-8

Takemoto-Kuno Y, Suzuki K, Nakamura S, Satoh H, Ohtsubo K (2006) Soluble starch synthase I effects differences in amylopectin structure between indica and japonica rice varieties. J Agric Food Chem 54(24):9234-40

Thivend P, Mercier C, Guilbot A (1965) Anwendung der Gluco-Amylase zur Stärkebestimmung. Stärke 17(9):278-83

Tsuji H, Taoka K, Shimamoto K (2013) Florigen in rice: complex gene network for florigen transcription, florigen activation complex, and multiple functions. Curr Opin Plant Biol 16(2):228-35

Tuncel A, Kawaguchi J, Ihara Y, Matsusaka H, Nishi A, Nakamura T, Kuhara S, Hirakawa H, Nakamura Y, Cakir B, Nagamine A, Okita TW, Hwang SK, Satoh H (2014) The rice endosperm ADP-glucose pyrophosphorylase large subunit is essential for optimal catalysis and allosteric regulation of the heterotetrameric enzyme. Plant Cell Physiol 55(6):1169-83

Vandesompele J, De Preter K, Pattyn F, Poppe B, Van Roy N, De Paepe A, Speleman F (2002) Accurate normalization of real-time quantitative RT-PCR data by geometric averaging of multiple internal control genes. Genome Biol 18: Research0034

Wang JC, Xu H, Zhu Y, Liu QQ, Cai XL (2013) OsbZIP58, a basic leucine zipper transcription factor, regulates starch biosynthesis in rice endosperm. J Exp Bot 64(11):3453-66

Yamakawa H, Hirose T, Kuroda M, Yamaguchi T (2007) Comprehensive expression profiling of rice grain filling-related genes under high temperature using DNA microarray. Plant Physiol 144(1):258-77

Yamamoto K, Sawada S, Onogaki T (1973) Properties of rice starch prepared by alkali method with various conditions. Denpun Kagaku 20(3):99-104 (in Japanese)

Yang J, Zhang J, Huang Z, Wang Z, Zhu Q, Liu L (2002) Correlation of cytokinin levels in the endosperms and roots with cell number and cell division activity during endosperm development in rice. Ann Bot 90(3):369-77

Yoshida S, Hara T (1977) Effects of air temperature and light on grain filling of an Indica and a Japonica rice (Oryza sativa L.) under controlled environmental conditions. Soil Sci Plant Nutr 23(1):93-107

\section{Submit your manuscript to a SpringerOpen ${ }^{\mathcal{O}}$ journal and benefit from:}

- Convenient online submission

- Rigorous peer review

- Immediate publication on acceptance

- Open access: articles freely available online

- High visibility within the field

- Retaining the copyright to your article

Submit your next manuscript at $\gg$ springeropen.com 